\title{
OPTIMAL CRITERIA FOR BLOWUP OF RADIAL AND $N$-SYMMETRIC SOLUTIONS OF CHEMOTAXIS SYSTEMS
}

\author{
PIOTR BILER, GRZEGORZ KARCH, AND JACEK ZIENKIEWICZ
}

\begin{abstract}
A simple proof of concentration of mass equal to $8 \pi$ for blowing up $N$-symmetric solutions of the Keller-Segel model of chemotaxis in two dimensions with large $N$ is given. Moreover, a criterion for blowup of solutions in terms of the radial initial concentrations, related to suitable Morrey spaces norms, is derived for radial solutions of chemotaxis in several dimensions. This condition is, in a sense, complementary to the one guaranteeing the global-intime existence of solutions.
\end{abstract}

\section{INTRODUCTION}

We consider in this paper the classical parabolic-elliptic Keller-Segel model of chemotaxis in $d \geq 2$ space dimensions

$$
\begin{array}{r}
u_{t}-\Delta u+\nabla \cdot(u \nabla v)=0, \\
\Delta v+u=0,
\end{array}
$$

supplemented with a nonnegative initial condition

$$
u(x, 0)=u_{0}(x) \geq 0 .
$$

Here for $(x, t) \in \mathbb{R}^{d} \times[0, T)$, the function $u=u(x, t) \geq 0$ denotes the density of the population of microorganisms, $v=v(x, t)$ — the density of the chemical secreted by themselves that attracts them and makes them to aggregate. The system (1.1)(1.2) also models the gravitational attraction of particles in astrophysical models, see $[2,3]$.

Date: August 26, 2018.

2010 Mathematics Subject Classification. 35Q92, 35B44, 35K55.

Key words and phrases. chemotaxis, blowup of solutions.

The authors thank the referees for many pertinent remarks that permitted them to improve the presentation of results. The preparation of the paper was supported by the NCN grant 2013/09/B/ST1/04412 (the first and the second authors). The third author was also supported by the grant DEC-2012/05/B/ST1/00692. 
As it is well known, cf. e.g. $[9,1]$, the total mass of the initial condition

$$
M=\int_{\mathbb{R}^{d}} u_{0}(x) \mathrm{d} x
$$

is conserved in time, i.e. $\int_{\mathbb{R}^{d}} u(x, t) \mathrm{d} x=\int_{\mathbb{R}^{d}} u_{0}(x) \mathrm{d} x=M$ for all $t \in\left[0, T_{\mathrm{b}}\right)$, and this is the critical quantity for the global-in-time existence of nonnegative solutions in the two-dimensional case. Namely, if $M \leq 8 \pi$, then solutions of (1.1)-(1.3) (with $u_{0}$ - a finite nonnegative measure) exist for all $t \geq 0$. For the local-in-time existence, it should be assumed that all the atoms of the finite measure $u_{0}$ are of mass less than $8 \pi$, see $[9,1,8]$.

When $M>8 \pi$, nonnegative solutions blow up in a finite time, and for radially symmetric solutions mass equal to $8 \pi$ concentrates at the origin at the blowup time, see [16, Ch. 11], [4], resp. The multidimensional case is different: there are solutions of the chemotaxis system with arbitrarily small $M>0$ that cease to exist after a finite time elapsed, see for instance $[3,10,5]$.

Global-in-time solutions of the chemotaxis system have been constructed in various functional spaces (including, e.g., finite Radon measures if $d=2$, and Lebesgue $L^{p}$, Marcinkiewicz weak $L^{p}$ spaces with $p=d / 2$, Besov and Morrey spaces if $d \geq 3$ ), cf. for instance $[1,2,12]$, under smallness conditions on norms of $u_{0}$ in a relevant space which is critical for (1.1)-(1.2). Here "critical" means that solutions obey the same scaling property as the norms in those spaces, cf. [12] for more detailed explanations.

First, we show in the present work that the radial concentration of data is the critical quantity for the finite time blowup of nonnegative radial solutions of (1.1)(1.3). Here, we define the radial concentration by the formula

$$
\left\|u_{0}\right\| \equiv \sup _{R>0} R^{2-d} \int_{\{|x| \leq R\}} \psi\left(\frac{x}{R}\right) u_{0}(x) \mathrm{d} x
$$

with a fixed radial nonnegative, piecewise $\mathcal{C}^{2}$ function $\psi$ supported on the unit ball, such that $\psi(0)=1$. Clearly, those quantities for such weight functions $\psi$ are comparable, so we fix in the following $\psi(x)=\left(1-|x|^{2}\right)_{+}^{2}$, see (2.2) below. 
Here, one should recall that the homogeneous Morrey space $M^{p}\left(\mathbb{R}^{d}\right), 1<p<\infty$, is defined as the set of all locally integrable functions $f$ that satisfy

$$
\|f\|_{M^{p}}=\sup _{x_{0} \in \mathbb{R}^{d}, R>0} R^{d(1 / p-1)} \int_{\left\{\left|y-x_{0}\right| \leq R\right\}}|f(y)| \mathrm{d} y<\infty .
$$

Of course, for $d \geq 3$ and $p=d / 2$, the norm in $M^{d / 2}\left(\mathbb{R}^{d}\right)$ (relevant to the theory of existence of local-in-time solutions) dominates the radial concentration (1.5): $\left\|u_{0}\right\|_{M^{d / 2}} \geq R^{2-d} \int_{\{|x| \leq R\}} u_{0}(x) \mathrm{d} x$ for each $R>0$, but, in fact, for radially symmetric $u_{0}$ both quantities $\left\|u_{0}\right\|_{M^{d / 2}}$ and $\left\|u_{0}\right\|$ are equivalent.

The criticality of the radial concentration (1.5) means that for initial data with small $\left\|u_{0}\right\|$ solutions exist indefinitely in time (cf. $[2,12]$ ), while our result stated in Theorem 1.1 below shows that for sufficiently big $\left\|u_{0}\right\|$ regular solutions cease to exist in a finite time. This result seems to be new for $d \geq 3$ although related criteria appeared in, e.g., [3] and [5]. They have been, however, formulated in terms of "global quantities" like the second moment $\int|x|^{2} u_{0}(x) \mathrm{d} x$ while (1.5) is a local quantity, and its definition does not require supplementary properties of $u_{0}$ like $\int|x|^{2} u_{0}(x) \mathrm{d} x<\infty$.

The proof of our first result (contained in the following theorem) on the occurrence of radially symmetric blowup for $d \geq 2$ does not involve "global quantities", and its idea is astonishingly simple.

Theorem 1.1 (Blowup of radial solutions with large concentration). For each $d \geq 2$ there exists a constant $C_{d}>0$ such that if $u_{0} \in L^{1}\left(\mathbb{R}^{d}\right)$ is a radially symmetric function and $R^{2-d} \int_{\{|x| \leq R\}} \psi\left(\frac{x}{R}\right) u_{0}(x) \mathrm{d} x>C_{d}$ for some $R>0$, then the solution $u$ of problem (1.1)-(1.3) blows up in a finite time.

Remark 1.2. Note that for $d=2$ we recover the well-known result: if $M>C_{2}=8 \pi$, then the solution of (1.1)-(1.3) blows up in a finite time, see the end of the proof of Theorem 1.1 below. In fact, this proof (involving a local moment of the solution) extends to the two-dimensional case $\left(x \in \mathbb{R}^{2}\right.$ ) of arbitrary (not necessarily radially symmetric) nonnegative solutions, cf. $[13,11,6]$ for similar arguments. Some 
improvements of the results in Theorem 1.1 (with direct relations to the critical values of Morrey norms and with quite different proofs) are in [7].

In our second result, we limit ourselves to the Cauchy problem (1.1)-(1.3) on the plane, and we show a concentration at the origin of mass equal exactly to $8 \pi$ for some solutions. This result is known in the radially symmetric case; our proof allows us to deal with a larger class of solutions, and is conceptually much simpler than existing ones. An analysis of the chemotaxis system in bounded planar domains leads to blowups at interior and boundary points, see [16, Th. 1.1], and to the quantization of mass $8 k \pi, k \in \mathbb{N}$, at the interior blowup points for solutions with finite free energy, see $[16$, Th. 1.2, Th. 15. 1]. The proofs of those results in [16, Ch. 11-15] rely on subtle estimates of the free energy for (1.1)-(1.2) and various functional inequalities of Gagliardo-Nirenberg-Sobolev type. For an earlier approach to different versions of conjectures on concentration we refer the reader to $[15$, p. 23-24]. Compare also [4] for the radially symmetric case.

Our proof for radially symmetric solutions with

$$
u_{0}\left(z \mathrm{e}^{i \vartheta}\right)=u_{0}(z), \quad \vartheta \in \mathbb{R}
$$

extends to the case of $N$-symmetric solutions with sufficiently large $N$, i.e. those with the initial data satisfying

$$
u_{0}\left(z \mathrm{e}^{i k \frac{2 \pi}{N}}\right)=u_{0}(z), \quad k \in \mathbb{N}
$$

with the natural identification $\mathbb{R}^{2} \ni x \leftrightarrow z \in \mathbb{C}$. Note that by the uniqueness of nonnegative solutions, the solution $u(x, t)$ with $u_{0}$ satisfying (1.7) is radial, and for that satisfying (1.8) $u$ is $N$-symmetric for each admissible $t: u\left(z \mathrm{e}^{i \frac{k}{N} \pi}, t\right)=u(z, t)$. The interest in such solutions is related to the problem of study of certain bilinear integrals involving derivatives of the fundamental solution of Laplacian.

Moreover, we are motivated by results in [14], where 2-symmetric distributions have been considered, see Remark 1.5 below for more information.

Theorem 1.3 (Blowup with $8 \pi$ concentration of mass). Assume that the initial condition $0 \leq u_{0} \in L^{1}\left(\mathbb{R}^{2}\right)$ is $N$-symmetric in the sense of equation (1.8) and such 
that $\int u_{0}(x) \mathrm{d} x=M>8 \pi$. Let $u(x, t)$, for $x \in \mathbb{R}^{2}$ and $t<T_{\mathrm{b}}$, be the corresponding classical solution of problem (1.1)-(1.3) which cannot be continued past the blowup time $t=T_{\mathrm{b}}$. If $N$ is sufficiently large so that $M / N$ is small enough, then

$$
\limsup _{t \rightarrow T_{\mathrm{b}}, R \rightarrow 0} \int_{\{|x| \leq R\}} u(x, t) \mathrm{d} x \leq 8 \pi .
$$

In fact, $N$-symmetric solutions blow up with the concentration of mass equal to $8 \pi$.

Corollary 1.4. Under the assumptions of Theorem 1.3, if moreover, $\int u_{0}(x)|x|^{2} \mathrm{~d} x<$ $\infty$, then

$$
\lim _{R \rightarrow 0} \lim _{t \rightarrow T_{\mathrm{b}}} \int_{\{|x| \leq R\}} u(x, t) \mathrm{d} x=8 \pi .
$$

In the proof of Theorem 1.3, we use simple (but rather subtle) techniques of weight functions and scalings. The core of our analysis consists in uniform (with respect to initial data) estimates on a blowup time (see Proposition 3.1) and on the uniform spread (or decay) of mass for symmetric initial conditions (see Proposition 3.9). The proofs of these two propositions is much shorter is the radially symmetric case, which we emphasize below. Moreover, we use systematically the well-known rescaling of the system: for each $\lambda>0$ and each solution $u$ of (1.1)-(1.2) of mass $M$ the function

$$
u_{\lambda}(x, t)=\lambda^{2} u\left(\lambda x, \lambda^{2} t\right)
$$

is also a solution, with its mass equal to $M$.

Corollary 1.4 is a direct consequence of Theorem 1.3 combined with results proved in [8], see the end of Section 3.

Remark 1.5. The authors of [14] suggested how to construct solutions of the KellerSegel system that blow up with the quantized concentration of mass $M=16 \pi$. In view of Theorem 1.3, their data cannot be $N$-symmetric with large $N \gg 2$. 
Notations. The integrals with no integration limits are understood as over the whole space $\mathbb{R}^{d}: \quad \int=\int_{\mathbb{R}^{d}}$. The letter $C$ denotes various constants which may vary from line to line but they are independent of solutions. As usual, $\sigma_{d}=\frac{2 \pi^{d / 2}}{\Gamma(d / 2)}$ denotes the area of the unit sphere in $\mathbb{R}^{d}$.

\section{Proof OF BLOWUP OF RADIAL SOLUTIONS}

We begin with two elementary observations which will be used in the proof of Theorem 1.1.

Lemma 2.1. Let $u \in L_{\mathrm{loc}}^{1}\left(\mathbb{R}^{d}\right)$ be a radially symmetric function, such that $v=$ $E_{d} * u$ with $E_{2}(x)=-\frac{1}{2 \pi} \log |x|$ and $E_{d}(x)=\frac{1}{(d-2) \sigma_{d}}|x|^{2-d}$ for $d \geq 3$, solves the Poisson equation $\Delta v+u=0$. Then

$$
\nabla v(x) \cdot x=-\frac{1}{\sigma_{d}}|x|^{2-d} \int_{\{|y| \leq|x|\}} u(y) \mathrm{d} y .
$$

Proof. By the Gauss formula, we have for the distribution function $M$ of $u$

$$
M(R) \equiv \int_{\{|y| \leq R\}} u(y) \mathrm{d} y=-\int_{\{|y|=R\}} \nabla v(y) \cdot \frac{y}{|y|} \mathrm{d} S .
$$

Thus, for the radial function $\nabla v(x) \cdot \frac{x}{|x|}$ and $|x|=R$, we obtain the identity

$$
\nabla v(x) \cdot x=\frac{1}{\sigma_{d}} R^{2-d} \int_{\{|y|=R\}} \nabla v(y) \cdot \frac{y}{|y|} \mathrm{d} S=-\frac{1}{\sigma_{d}} R^{2-d} M(R) .
$$

Lemma 2.2. If $\omega \in L_{\mathrm{loc}}^{1}\left(\mathbb{R}^{d}\right)$ is a radially symmetric function and $M(R)=$ $\int_{\{|x| \leq R\}} \omega(x) \mathrm{d} x-$ its distribution function, then

$$
\int_{\{|x| \leq R\}} \omega(x) M(|x|) \mathrm{d} x=\frac{1}{2} M(R)^{2} .
$$


Proof. Since $\omega$ is radial, it satisfies for $|x|=R$ the equality $\omega(x)=\frac{1}{\sigma_{d}} R^{1-d} M^{\prime}(R)$.

Thus, using the polar coordinates, we obtain

$$
\begin{aligned}
\int_{\{|x| \leq R\}} \omega(x) M(|x|) \mathrm{d} x & =\sigma_{d} \int_{0}^{R} \frac{1}{\sigma_{d}} r^{1-d} M^{\prime}(r) M(r) r^{d-1} \mathrm{~d} r \\
& =\int_{0}^{R} M^{\prime}(r) M(r) \mathrm{d} r=\frac{1}{2} M(R)^{2} .
\end{aligned}
$$

Proof of Theorem 1.1. We will derive a differential inequality for the quantity

$$
w_{R}(t)=\int \psi_{R}(x) u(x, t) \mathrm{d} x
$$

with the scaled weight function $\psi_{R}$ supported on the ball $\{|x| \leq R\}$

$$
\psi(x)=\left(1-|x|^{2}\right)^{2} \mathbb{I}_{\{|x| \leq 1\}} \quad \text { and } \quad \psi_{R}(x)=\psi\left(\frac{x}{R}\right) \quad \text { with } \quad R>0 .
$$

The function $\psi \in \mathcal{C}^{1}\left(\mathbb{R}^{d}\right)$ has piecewise continuous and bounded second derivatives

$$
\begin{aligned}
& \nabla \psi(x)=-4 x\left(1-|x|^{2}\right) \mathbb{1}_{\{|x| \leq 1\}}=-4 x \psi(x)^{1 / 2}, \\
& \Delta \psi(x)=\left(-4 d+4(d+2)|x|^{2}\right) \mathbb{I}_{\{|x| \leq 1\}} .
\end{aligned}
$$

Observe that $\psi$ satisfies the relation

$$
\Delta \psi(x) \geq-\frac{(d+2)^{2}}{2} \psi(x),
$$

which is seen from the elementary inequality for the quadratic polynomial

$$
-4 d+4(d+2) s \geq-\frac{(d+2)^{2}}{2}(1-s)^{2},
$$

equivalent to $\left(s-\frac{d-2}{d+2}\right)^{2} \geq 0$, applied to $0 \leq s \leq 1$.

Now, using equation (1.1), integrations by parts and applying relations (2.2)(2.4), we obtain

$$
\begin{aligned}
\frac{\mathrm{d}}{\mathrm{d} t} w_{R}(t) & =\int \Delta \psi_{R}(x) u(x, t) \mathrm{d} x+\int u(x, t) \nabla v(x, t) \cdot \nabla \psi_{R}(x) \mathrm{d} x \\
(2.5) & \geq R^{-2}\left(-\frac{(d+2)^{2}}{2} \int \psi_{R}(x) u(x, t) \mathrm{d} x-4 \int u(x, t)(\nabla v(x, t) \cdot x)\left(\psi_{R}(x)\right)^{1 / 2} \mathrm{~d} x\right) .
\end{aligned}
$$

Thus, by Lemma 2.1, we get

$$
\begin{aligned}
R^{2} \frac{\mathrm{d}}{\mathrm{d} t} w_{R}(t) & \geq-\frac{(d+2)^{2}}{2} w_{R}(t)+\frac{4}{\sigma_{d}} \int u(x, t) M(|x|, t)|x|^{2-d} \psi_{R}(x)^{1 / 2} \mathrm{~d} x \\
& \geq-\frac{(d+2)^{2}}{2} w_{R}(t)+\frac{4}{\sigma_{d}} R^{2-d} \int \psi_{R}(x) u(x, t) M(|x|, t) \mathrm{d} x
\end{aligned}
$$


because $\psi_{R}(x)=0$ for $|x| \geq R$, and $\psi_{R}(x) \leq \psi_{R}(x)^{1 / 2}$.

Now, note that obviously

$$
M(R, t)=\int_{\{|y| \leq R\}} u(y, t) \mathrm{d} y \geq \int_{\{|y| \leq R\}} \psi_{R}(y) u(y, t) \mathrm{d} y .
$$

Hence, applying Lemma 2.2 to the radial function $\omega(x)=\psi_{R}(x) u(x, t)$, we obtain

$$
\int \psi_{R}(x) u(x, t) M(|x|, t) \mathrm{d} x \geq \frac{1}{2}\left(\int \psi_{R}(x) u(x, t) \mathrm{d} x\right)^{2} .
$$

Thus, as a consequence of inequality (2.6), we arrive at

$$
R^{2} \frac{\mathrm{d}}{\mathrm{d} t} w_{R}(t) \geq-\frac{(d+2)^{2}}{2} w_{R}(t)+\frac{2}{\sigma_{d}} R^{2-d} w_{R}(t)^{2} .
$$

Now, it is clear from (2.7) that if

$$
R^{2-d} w_{R}(0)>\frac{(d+2)^{2}}{2} /\left(\frac{2}{\sigma_{d}}\right)=(d+2)^{2} \frac{\sigma_{d}}{4} \equiv C_{d},
$$

then $-\frac{(d+2)^{2}}{2} w_{R}(0)+\frac{2}{\sigma_{d}} R^{2-d} w_{R}(0)^{2} \equiv \delta>0$. Since the right-hand side of (2.7) is an increasing function of $w_{R}$, we have $\frac{\mathrm{d}}{\mathrm{d} t} w_{R}(t) \geq \delta>0$. As a consequence, the function $w_{R}(t)$ becomes greater than $M=\int u(x, t) \mathrm{d} x$ in a finite time which is a contradiction with the existence of nonnegative, mass conserving solutions.

Finally, observe that if $d=2$ the conditions $M>C_{2} \equiv 8 \pi,\left\|u_{0}\right\|>8 \pi$ and $w_{R}(0)>8 \pi$ for $R>0$ sufficiently large are equivalent.

Similarly, if $d \geq 3$, the conditions $\left\|u_{0}\right\|>C_{d}$ and $R^{2-d} w_{R}(0)>C_{d}$ for some $R>0$ are equivalent.

\section{Blowup With $8 \pi$ CONCENTRATION OF MASs}

The proof of Theorem 1.3, saying that a solution to problem (1.1)-(1.3) on the whole plane $\mathbb{R}^{2}$ with $M>8 \pi$ concentrates at the origin with mass not exceeding $8 \pi$ at the blowup time, is based on two auxiliary results: on a uniform estimate of the blowup time (Proposition 3.1) and on a uniformly slow spread of mass over annuli in $\mathbb{R}^{2}$ (Proposition 3.9).

3.1. Uniform blowup time. In the following proposition, we show that the blowup time of a solution to problem (1.1)-(1.3) can be estimated from above by a number which depends only on an amount of $u_{0}$ concentrated in the unit ball. 
Proposition 3.1. Let $\varepsilon>0$ and $\gamma>0$ be arbitrary and fixed. Suppose that $u=u(x, t)$ is a solution of problem (1.1)-(1.3) with an initial datum satisfying

$$
0 \leq u_{0} \in L^{1}\left(\mathbb{R}^{2}\right) \quad \text { and } \int_{\{|x| \leq \gamma\}} u_{0}(x) \mathrm{d} x \geq 8 \pi+\varepsilon .
$$

Then $u(x, t)$ blows up in a finite time $t=T_{\mathrm{b}} \leq \gamma^{2} T(M, \varepsilon)$, where $T(M, \varepsilon)>0$ depends on $M=\int u_{0}(x) \mathrm{d} x>8 \pi$ and $\varepsilon$, only.

Remark 3.2. We introduce the parameter $\gamma>0$ in Proposition 3.1 to simplify the notation in the proof. In fact, assuming the property stated in the proposition for $\gamma=1$, we obtain immediately this property for each other $\gamma>0$ by the rescaling $u \mapsto u_{\gamma^{-1}}=\gamma^{-2} u\left(\gamma^{-1} x, \gamma^{-2} t\right)$.

Remark 3.3. Observe that for an initial condition $u_{0}$ with its support in the unit ball, this proposition holds true by the standard second moment argument (cf. e.g. $[3,9])$ based on the identity

$$
\frac{\mathrm{d}}{\mathrm{d} t}\left(\int|x|^{2} u(x, t) \mathrm{d} x\right)=\frac{1}{2 \pi} M(8 \pi-M)<0,
$$

which implies that a nonnegative solution $u(x, t)$ ceases to exist at a moment of time estimated from above by the number $2 \pi(M(M-8 \pi))^{-1} \int|x|^{2} u_{0}(x) \mathrm{d} x$. Now, it suffices to notice that $\int|x|^{2} u_{0}(x) \mathrm{d} x \leq M$ for supp $u_{0} \subset\{|x| \leq 1\}$ and choose $\varepsilon=M-8 \pi$.

Remark 3.4. Proposition 3.1 has been already proved in this paper in the radially symmetric case. Indeed, it is sufficient to apply inequality (2.7) with $d=2$ and a suitable $R>0$ :

$$
R^{2} \frac{\mathrm{d}}{\mathrm{d} t} w_{R}(t) \geq-8 w_{R}(t)+\frac{1}{\pi} w_{R}(t)^{2}
$$

to the function $w_{R}$ defined by relations $(2.1)-(2.2)$. We use this inequality with $R=2^{1 / 2}(2+16 \pi / \varepsilon)^{1 / 2}$. By a direct calculation using the assumption on $u_{0}$, we obtain

$$
w_{R}(0) \geq\left(1-\frac{1}{R^{2}}\right)^{2} \int_{\{|x| \leq 1\}} u_{0}(x) \mathrm{d} x \geq\left(1-\frac{1}{R^{2}}\right)^{2}(8 \pi+\varepsilon) \geq 8 \pi+\frac{\varepsilon}{2} .
$$


Thus, analogously as at the end of the proof of Theorem 1.1, the function $w_{R}(t)$ becomes greater than $M$ in a finite time which can be estimated from above by a quantity depending on $M$ and $\varepsilon$, only.

Remark 3.5. We cannot directly apply the local moment method developed in [6] to show Proposition 3.1 for general initial conditions, analogously as in the radial case discussed in Remark 3.4. This is due to the fact that blowup results in [6] are proved for each initial datum $u_{0}$ such that $M=\int u_{0}(x) \mathrm{d} x>8 \pi$ which, moreover, has a small mass outside a ball. In fact, by methods of this work, we can remove that extra assumption from results proved in [6].

One may summarize Remarks $3.3-3.5$ by saying that the main problem in proving Proposition 3.1 consists in controlling a large mass of a solution which is outside of the unit ball. To show this proposition, we study (as in the previous section) the time evolution of the function

$$
w(t)=w_{1}(t)=\int \psi(x) u(x, t) \mathrm{d} x
$$

and a solution $u(x, t)$ blows up at certain $T_{\mathrm{b}}$ if there exists $T \geq T_{\mathrm{b}}$ such that $w(T)=$ $M$. Here, besides inequalities (2.3)-(2.4), we will use the following elementary estimates for the weight function $\psi=\psi_{1}(x)=\left(1-|x|^{2}\right)^{2} \mathbb{I}_{\{|x| \leq 1\}}$ :

$$
\begin{aligned}
|\psi(x)-1| & \leq B|x|^{2}, \\
|\nabla \psi(x)-\nabla \psi(y)+4(x-y)| & \leq B \delta|x-y| \text { for all }|x|,|y| \leq \delta, \\
|(x-y) \cdot(\nabla \psi(x)-\nabla \psi(y))| & \leq B \min \left\{|x-y|^{2},|x-y|\right\},
\end{aligned}
$$

valid for each fixed constant $0<\delta<1$, some constant $B \geq 1$ independent of $\delta$, and all $x, y \in \mathbb{R}^{2}$.

First, let us prove an auxiliary result concerning the function $w(t)$.

Lemma 3.6. Given $\varepsilon \in(0, M-8 \pi]$, define the parameters

$$
\eta=\eta(\varepsilon)=\frac{\varepsilon}{100 M^{2} B}, \quad \alpha=\frac{1}{100 M B}, \quad \lambda=\lambda(\varepsilon)=\frac{100 M^{2} B}{\varepsilon}+1,
$$


where $B \geq 1$ is a constant satisfying (3.2)-(3.4). Assume that

$$
w(0) \geq 8 \pi+\frac{\varepsilon}{2}
$$

Suppose that there exists $T \in\left(0, T_{\mathrm{b}}\right]$ such that for all $t \in[0, T)$ we have the estimate

$$
\int_{\{\eta \leq|x| \leq \lambda\}} u(x, t) \mathrm{d} x<\varepsilon \alpha .
$$

Then, for all $t \in[0, T]$, the inequality $\frac{\mathrm{d}}{\mathrm{d} t} w(t) \geq \frac{1}{100} \varepsilon$ holds true. In particular, the estimate $T \leq 100 M / \varepsilon$ follows.

Proof. Applying inequality (3.4) and then assumption (3.7), we have for our choice of $\eta, \alpha, \lambda$

$$
\int_{\{|y| \leq \eta\}} \int_{\{\eta \leq|x| \leq \lambda\}} u(x, t) u(y, t) \frac{|(x-y) \cdot(\nabla \psi(x)-\nabla \psi(y))|}{|x-y|^{2}} \mathrm{~d} x \mathrm{~d} y \leq B M \varepsilon \alpha=\frac{\varepsilon}{100},
$$

and again by (3.4) since $\eta<1$

$$
\int_{\{|y| \leq \eta\}} \int_{\{\lambda \leq|x|\}} u(x, t) u(y, t) \frac{|(x-y) \cdot(\nabla \psi(x)-\nabla \psi(y))|}{|x-y|^{2}} \mathrm{~d} x \mathrm{~d} y \leq \frac{M^{2} B}{\lambda-1}=\frac{\varepsilon}{100} .
$$

Moreover, by (3.3) and elementary calculations

$$
\begin{aligned}
& \int_{\{|y| \leq \eta\}} \int_{\{|x| \leq \eta\}} u(x, t) u(y, t) \frac{(x-y) \cdot(\nabla \psi(x)-\nabla \psi(y))}{|x-y|^{2}} \mathrm{~d} x \mathrm{~d} y \\
\geq & w(t)^{2}-2 w(t) \int_{\{\eta \leq|x| \leq 1\}} \psi(x) u(x, t) \mathrm{d} x-\frac{1}{25} \varepsilon \\
\geq & w(t)^{2}-2 M \varepsilon \alpha-\frac{1}{25} \varepsilon \\
= & w(t)^{2}-\frac{1}{50} \varepsilon-\frac{1}{25} \varepsilon \geq w(t)^{2}-\frac{1}{10} \varepsilon .
\end{aligned}
$$


Hence, repeating the above estimate with $x$ replaced by $y$, and using (2.4) with $d=2$, we obtain

$$
\begin{aligned}
\frac{\mathrm{d}}{\mathrm{d} t} w(t) & =\int \psi(x) \Delta u(x, t) \mathrm{d} x-\frac{1}{2 \pi} \iint u(x, t) u(y, t) \frac{x-y}{|x-y|^{2}} \cdot \nabla \psi(x) \mathrm{d} x \mathrm{~d} y \\
& =\int \Delta \psi(x) u(x, t) \mathrm{d} x-\frac{1}{4 \pi} \iint u(x, t) u(y, t) \frac{x-y}{|x-y|^{2}} \mathrm{~d} x \mathrm{~d} y \cdot(\nabla \psi(x)-\nabla \psi(y)) \mathrm{d} x \mathrm{~d} y \\
& \geq-8 w(t)+\frac{1}{\pi}\left(w(t)^{2}-\frac{\varepsilon}{10}\right) \\
& =\frac{1}{\pi} w(t)(w(t)-8 \pi)-\frac{1}{10 \pi} \varepsilon \geq \frac{1}{2 \pi} \varepsilon-\frac{1}{10 \pi} \varepsilon \geq \frac{1}{4 \pi} \varepsilon
\end{aligned}
$$

as long as $w(t)$ is increasing. Now, Lemma 3.6 follows by assumption (3.6).

Since we cannot have the estimate $w(T)>M$, integrating the differential inequality $\frac{\mathrm{d}}{\mathrm{d} t} w(t) \geq \frac{1}{100} \varepsilon$ we obtain the upper bound $T \leq 100 \mathrm{M} / \varepsilon$.

Now, we define a certain property of problem (1.1)-(1.3).

Definition 3.7. Fix $\varepsilon>0$ and $\gamma>0$. Problem (1.1)-(1.3) is said to have the property $\mathcal{I}_{\varepsilon}$ if each of its solutions corresponding to an initial datum satisfying

$$
0 \leq u_{0} \in L^{1}\left(\mathbb{R}^{2}\right) \quad \text { and } \int_{\{|x| \leq \gamma\}} u_{0}(x) \mathrm{d} x \geq 8 \pi+\varepsilon
$$

blows up not later than at time $\gamma^{2} T(M, \varepsilon)$, with the parameter $T(M, \varepsilon)>0$ depending on $M=\int u_{0}(x) \mathrm{d} x>8 \pi$ and $\varepsilon$, only.

Let us first notice elementary facts concerning the property $\mathcal{I}_{\varepsilon}$.

Remark 3.8. The parameter $\gamma>0$ can be easily removed from this definition by the usual rescaling, cf. Remark 3.2. Problem (1.1)-(1.3) has the property $\mathcal{I}_{M-8 \pi}$, because then assumptions (3.10) with $\varepsilon=M-8 \pi$ mean that $u_{0}$ is supported on the ball of radius $\gamma$. Hence, it suffices to apply Remark 3.3. Obviously, there is no solution satisfying conditions (3.10) for $\varepsilon>M-8 \pi$. It is also easy to show that if problem (1.1)-(1.3) has the property $\mathcal{I}_{\varepsilon}$, then it has the property $\mathcal{I}_{\tilde{\varepsilon}}$ for each $\tilde{\varepsilon}>\varepsilon$. Proof of Proposition 3.1. By Definition 3.7, it suffices to show that problem (1.1)(1.3) has the property $\mathcal{I}_{\varepsilon}$ for all $\varepsilon>0$ and with a suitably chosen $\gamma>0$. To do this, we are going to prove the following two claims for each $\varepsilon>0$ with parameters $\alpha=\alpha(\varepsilon), \eta$, and $\lambda(\varepsilon)$ defined in (3.5). 
- Claim 1. Suppose that $\varepsilon$ and $M$ satisfy the conditions

$$
8 \pi+\varepsilon\left(1+\eta^{2} \alpha / 2\right)>M
$$

Then problem (1.1)-(1.3) has the property $\mathcal{I}_{\varepsilon}$.

- Claim 2. Suppose that

$$
8 \pi+\varepsilon\left(1+\eta^{2} \alpha / 2\right) \leq M
$$

and problem (1.1)-(1.3) has the property $\mathcal{I}_{\varepsilon\left(1+\eta^{2} \alpha / 2\right)}$. Then problem (1.1)(1.3) has the property $\mathcal{I}_{\varepsilon}$.

Let us first prove that Claims 1 and 2 imply the property $\mathcal{I}_{\varepsilon}$ for all $\varepsilon>0$. Obviously, inequality (3.11) holds true for $\varepsilon=M-8 \pi$. Thus, since $\alpha=\alpha(\varepsilon)$ is a continuous function of $\varepsilon$, inequality (3.11) holds true for all $\varepsilon \in\left(\varepsilon_{0}, M-8 \pi\right]$ with some $\varepsilon_{0}<M-8 \pi$, and problem (1.1)-(1.3) has the property $\mathcal{I}_{\varepsilon}$ in this range of $\varepsilon$ by Claim 1. Recalling Remark 3.8, define

$$
\varepsilon_{0}=\inf \left\{\varepsilon>0: \text { problem }(1.1)-(1.3) \text { has the property } \mathcal{I}_{\varepsilon}\right\} \text {, }
$$

and suppose that $\varepsilon_{0}>0$. By continuity, there exists $\varepsilon_{1}>0$, such that $\varepsilon_{1}(1+$ $\left.\eta^{2} \alpha\left(\varepsilon_{1}\right) / 2\right)=\varepsilon_{0}$. For every $\tilde{\varepsilon} \in\left(\varepsilon_{1}, \varepsilon_{0}\right)$, we have the alternative: either $\tilde{\varepsilon}$ satisfies inequality (3.11) or inequality (3.12). In both cases, either by Claim 1 or Claim 2, problem (1.1)-(1.3) has the property $\mathcal{I}_{\tilde{\varepsilon}}$. This is a contradiction with the definition $\varepsilon_{0}$ because $\tilde{\varepsilon}<\varepsilon_{0}$.

Now, we prove both Claims 1 and 2 simultaneously, and the scheme of the proof is the following. If assumption (3.11) is satisfied and if estimate (3.7) holds true for all $t \in\left[0, T_{\mathrm{b}}\right)$, the proof of Claim 1 is completed by Lemma 3.6. At the first point $t=T_{1}$, where estimate (3.7) fails, we obtain inequality (3.12). Hence, using the recurrence hypothesis of Claim 2 and a suitable rescaling of the whole problem, we obtain Claim 2.

Fix $\varepsilon \in(0, M-8 \pi)$. Let $\eta=\eta(\varepsilon), \alpha, \lambda(\varepsilon)$ be defined by (3.5). Set

$$
\gamma^{2}=\frac{\alpha \eta^{2} \varepsilon}{2 M B}
$$


and notice that $\gamma^{2}=\gamma^{2}(\varepsilon) \leq 1$. Suppose that $u_{0}$ satisfies conditions (3.10) with this value of $\gamma$. Thus, using inequality (3.2), we obtain

$$
\begin{aligned}
w(0) & \geq \int_{\{|x| \leq \gamma\}} \psi(x) u_{0}(x) \mathrm{d} x \\
& \geq \int_{\{|x| \leq \gamma\}} u_{0}(x) \mathrm{d} x-\int_{\{|x| \leq \gamma\}}|1-\psi(x)| u_{0}(x) \mathrm{d} x \\
& \geq 8 \pi+\varepsilon-B \int_{\{|x| \leq \gamma\}}|x|^{2} u_{0}(x) \mathrm{d} x \\
& \geq 8 \pi+\varepsilon-B \gamma^{2} M .
\end{aligned}
$$

Notice that, with our choice of $\gamma$ in (3.13), we have $M B \gamma^{2}<\varepsilon / 2$, thus, we obtain the inequality $w(0)>8 \pi+\varepsilon / 2$, which is the first assumption (3.6) of Lemma 3.6.

Next, we deal with the second assumption (3.7) of Lemma 3.6. Notice that if estimate (3.7) holds true for all $t \in\left[0, T_{\mathrm{b}}\right)$ then, by Lemma 3.6, we have the property $\mathcal{I}_{\varepsilon}$ with $\gamma$ defined in (3.13).

Suppose that estimate (3.7) does not hold for $t=0$. Then, by assumption (3.10) and the inequalities $\gamma<\eta$ and $\eta^{2} \leq 1 / 2$, we obtain

$$
\begin{aligned}
\int_{\{|x| \leq \lambda\}} u_{0}(x) \mathrm{d} x & =\int_{\{|x| \leq \gamma\}} u_{0}(x) \mathrm{d} x+\int_{\{\gamma \leq|x| \leq \lambda\}} u_{0}(x) \mathrm{d} x \\
& \geq 8 \pi+\varepsilon+\varepsilon \alpha \geq 8 \pi+\varepsilon\left(1+\eta^{2} \alpha / 2\right) .
\end{aligned}
$$

Notice that this inequality cannot be true under the condition (3.11) of Claim 1 , because then the total mass of $u_{0}$ would be greater than $M$. Thus, we have inequality (3.12) assumed in Claim 2. Suppose that the second assumption of Claim 2 is satisfied, namely, that each solution of problem (1.1)-(1.3) with an initial datum satisfying (3.10) with $\varepsilon$ replaced by $\varepsilon\left(1+\eta^{2} \alpha / 2\right)$ blows up at time estimated from above by $\lambda^{2} T\left(M, \varepsilon\left(1+\eta^{2} \alpha / 2\right)\right)$. Now, we rescale the solution as explained in Remark 3.2, to see that it suffices to choose $T(M, \varepsilon)=\gamma^{-2} \lambda^{2} T\left(M, \varepsilon\left(1+\eta^{2} \alpha / 2\right)\right)$. Since $\gamma, \lambda$ depend only on $M, \varepsilon$, we obtain the property $\mathcal{I}_{\varepsilon}$.

Now, consider the case when assumption (3.7) of Lemma 3.6 is not satisfied for some $t \in\left(0, T_{\mathrm{b}}\right)$. Thus, by continuity, there exists $T_{1} \in\left(0, T_{\mathrm{b}}\right)$ such that strict 
inequality (3.7) is satisfied for all $t \in\left[0, T_{1}\right)$, and for $t=T_{1}$ we have

$$
\int_{\{\eta \leq|x| \leq \lambda\}} u\left(x, T_{1}\right) \mathrm{d} x=\varepsilon \alpha .
$$

Hence, by Lemma 3.6, the function $w(t)$ is increasing for $t \leq T_{1}$, and by (3.14) we obtain

$$
w\left(T_{1}\right)=\int \psi(x) u\left(x, T_{1}\right) \mathrm{d} x \geq w(0) \geq 8 \pi+\varepsilon-\gamma^{2} M B .
$$

Now, the estimate

$$
\mathbb{1}_{\{|x| \leq \lambda\}} \geq \psi(x)+\left(1-\left(1-\eta^{2}\right)^{2}\right) \mathbb{1}_{\{\eta \leq|x| \leq \lambda\}} \geq \psi(x)+\eta^{2} \mathbb{1}_{\{\eta \leq|x| \leq \lambda\}}
$$

implies

$$
\begin{aligned}
\int_{\{|x| \leq \lambda\}} u\left(x, T_{1}\right) \mathrm{d} x & \geq \int \psi(x) u\left(x, T_{1}\right) \mathrm{d} x+\eta^{2} \int_{\{\eta<|x|<\lambda\}} u\left(x, T_{1}\right) \mathrm{d} x \\
& \geq 8 \pi+\varepsilon-\gamma^{2} B M+\eta^{2} \varepsilon \alpha \geq 8 \pi+\varepsilon\left(1+\alpha \eta^{2} / 2\right),
\end{aligned}
$$

because $\gamma^{2} B M \leq \eta^{2} \varepsilon \alpha / 2$.

Notice again, as above, that condition (3.11) of Claim 1 cannot be true. Thus, if we assume both conditions of Claim 2, then problem (1.1)-(1.3) with the initial condition $u\left(x, T_{1}\right)$ blows up not later than at time $t=\lambda^{2} T\left(M, \varepsilon\left(1+\alpha \eta^{2} / 2\right)\right)$. Then, problem (1.1)-(1.3) with the initial condition $u_{0}$ blows up at time estimated from above by

$$
\tilde{T}(M, \varepsilon)=T_{1}+\lambda^{2} T\left(M, \varepsilon\left(1+\alpha \eta^{2} / 2\right)\right) \leq 100 M / \varepsilon+\lambda^{2} T\left(M, \varepsilon\left(1+\alpha \eta^{2} / 2\right)\right) .
$$

Now, it suffices to rescale the problem by choosing $T(M, \varepsilon)=\gamma^{-2} \tilde{T}(M, \varepsilon)$.

\subsection{Uniform spread of mass in the cases of $N$-symmetry of initial data.}

We prove our second auxiliary result for $N$-symmetric nonnegative solutions with sufficiently large $N$.

Proposition 3.9. Let $0 \leq u_{0} \in L^{1}\left(\mathbb{R}^{2}\right)$ and $\int_{\{\delta \leq|x| \leq R\}} u_{0}(x) \mathrm{d} x \geq \varepsilon$ for some $\varepsilon>0$ and $0<\delta<R / 4<\infty$. If $u_{0}$ is an $N$-symmetric function (see (1.8)) where the integer $N>0$ is sufficiently large, i.e. $N \geq c M$ with a constant $c>0$ independent 
of $M$ and of $\delta$, then the solution $u$ of problem (1.1)-(1.3), as long as this exists, satisfies for $t>0$

$$
\int_{\left\{\frac{1}{2} \delta \leq|x| \leq 3 R\right\}} u(x, t) \mathrm{d} x \geq \varepsilon \exp (-C t),
$$

where $C=C(M, \varepsilon, \delta)>0$ depends only on $M, \delta, \varepsilon$, and is independent of $R$.

Proof. First, we assume that $\delta=1$ and $1<R<\infty$. Consider a weight function $\phi: \mathbb{R}^{+} \rightarrow \mathbb{R}^{+}$such that

$$
\phi(s)= \begin{cases}0 & \text { if } 0 \leq s \leq \frac{1}{2} \\ (2 s-1)^{2} & \text { if } \frac{1}{2} \leq s \leq \frac{3}{4} \\ 1 & \text { if } 1 \leq s \leq R \\ \left(1-\frac{s-R}{2 R}\right)^{2} & \text { if } 2 R \leq s \leq 3 R \\ 0 & \text { if } 3 R \leq s\end{cases}
$$

Such a function $\phi$ can be chosen increasing on $[0, R]$, decreasing on $[R, 3 R], \operatorname{supp} \phi \subset$ $[1 / 2,3 R]$, and piecewise $\mathcal{C}^{2}$, with its derivatives $\phi^{(k)}$ satisfying for $k=0,1,2$ :

$$
\left|\phi^{(k)}(s)\right| \leq \frac{C}{s^{k}} \quad \text { with a constant } C \text { independent of } R \text { and of } s>0 .
$$

We define, for the function $\Phi(x)=\phi(|x|)$, the moment function of the solution $u$ by $H(t)=\int \Phi(x) u(x, t) \mathrm{d} x$ that measures mass of $u$ contained in the annulus $\left\{\frac{1}{2} \leq|x| \leq 3 R\right\}$.

First, we present a particularly simple argument for radial solutions based on the identity from Lemma 2.1. For the evolution of $H$ we have the differential inequality

$$
\begin{aligned}
\frac{\mathrm{d}}{\mathrm{d} t} H(t) & =\int \Phi(x) \Delta u(x, t) \mathrm{d} x+\int u(x, t) \nabla v(x, t) \cdot \nabla \Phi(x) \mathrm{d} x \\
& =\int \Delta \Phi(x) u(x, t) \mathrm{d} x+\int u(x, t)(\nabla v(x, t) \cdot x) \frac{\phi^{\prime}(|x|)}{|x|} \mathrm{d} x \\
& \geq \int u(x, t)\left(\Delta \Phi(x)-\frac{1}{2 \pi} M(|x|, t) \frac{\left|\phi^{\prime}(|x|)\right|}{|x|}\right) \mathrm{d} x \\
& \geq-C(M) \int \Phi(x) u(x, t) \mathrm{d} x=-C(M) H(t)
\end{aligned}
$$

with a constant $C(M)$ independent of $R$. In the last inequality, we have used the bound

$$
\Delta \Phi(x)-\frac{1}{2 \pi} M \frac{1}{|x|}\left|\phi^{\prime}(|x|)\right| \geq-C(M) \Phi(x)
$$


which is valid since $\Delta \Phi(x) \geq C|x|^{-2}>0$ for $\frac{1}{2} \leq|x| \leq \frac{3}{4}$ and $\frac{5}{2} R \leq|x| \leq 3 R$, and because of estimates (3.17). Since by the assumption we have $H(0) \geq \varepsilon>0$, the above inequality yields the conclusion of Proposition 3.9 for radial solutions.

Now, we prove Proposition 3.9 under the $N$-symmetry assumption. Our goal is to derive again a differential inequality of the form $\frac{\mathrm{d}}{\mathrm{d} t} H(t) \geq-C H(t)$ with a suitably large constant $C$ depending only on $M$ and $N$, as was done in (3.18). Let us emphasize here that the crucial consequence of the $N$-symmetry assumption consists in some cancellations in the bilinear (w.r.t. $u$ ) integral $\int u \nabla v \cdot \nabla \Phi$ appearing in the first line of formula (3.18).

Let us decompose the gradient of the weight function $\Phi$ as

$$
\nabla \Phi=\mu+\nu
$$

with

$$
\operatorname{supp} \mu \subset\left\{\frac{1}{2} \leq|x| \leq 2\right\}
$$

and

$$
\operatorname{supp} \nu \subset\{R \leq|x| \leq 3 R\}
$$

We write $\frac{\mathrm{d}}{\mathrm{d} t} H(t)=I_{0}+I_{1}$, where $I_{0}=\int \Delta \Phi(x) u(x, t) \mathrm{d} x$ and

$$
I_{1}=-\iint u(x, t) u(y, t) \frac{x-y}{|x-y|^{2}} \cdot \nabla \Phi(x) \mathrm{d} x \mathrm{~d} y \equiv I_{1, \mu}+I_{1, \nu},
$$

according to (3.20)-(3.21). Further, given $A \geq 4$, we decompose the integral $I_{1, \mu}$ into the sum of integrals

$$
I_{1, \mu}=J_{1}+J_{2}+J_{3}
$$

with the integration domains

$$
\left\{\frac{1}{4} \leq|x| \leq A,|y| \geq A\right\}, \quad\left\{\frac{1}{4} \leq|x| \leq 2,|y| \leq \frac{1}{4}\right\}, \quad\left\{\frac{1}{4} \leq|x| \leq A, \frac{1}{4} \leq|y| \leq A\right\},
$$

respectively. Note that, in fact, $\operatorname{supp} \mu \subset \subset\left\{\frac{1}{4} \leq|x| \leq 2\right\}$. 
The integrals $J_{1}, J_{2}$ will be estimated rather crudely. Using the property (3.20) we have the following bound for the integral $J_{1}$ :

$$
\begin{aligned}
J_{1} & =-\int_{\left\{\frac{1}{2} \leq|x| \leq 2\right\}} \int_{\{|y| \geq A\}} u(x, t) u(y, t) \frac{x-y}{|x-y|^{2}} \cdot \mu(x) \mathrm{d} x \mathrm{~d} y \\
& \geq-\frac{2 C}{A} M \int_{\left\{\frac{1}{2} \leq|x| \leq 2\right\}} u(x, t) \mathrm{d} x \\
& \geq-\frac{2 C M}{A}\left(\int_{\left\{\frac{1}{2} \leq|x| \leq 2\right\}} u \Delta \Phi+C \int_{\left\{\frac{1}{2} \leq|x| \leq 2\right\}} u \Phi\right),
\end{aligned}
$$

since for $|x| \leq 2$ and $4 \leq A \leq|y|$ the relation $|x-y| \geq \frac{1}{2} A$ holds and, moreover, $1 \leq \Delta \Phi(x)+C \Phi(x)$ for each $\frac{1}{2} \leq|x| \leq 2$. Finally, let us define $A=4 C M$.

Now, observe that for each $\varrho>0$ there exists a constant $C_{\varrho}$ such that the both "weights" $|\mu|$ and $|\mu|^{\frac{1}{2}}$ are bounded by $\Delta \Phi$ and $\Phi$, i.e. the inequality

$$
|\mu(x)|+|\mu(x)|^{\frac{1}{2}} \leq \varrho \Delta \Phi(x)+C_{\varrho} \Phi(x)
$$

holds. This inequality, together with $|x-y| \geq \frac{1}{4}$ and (3.20), leads to the estimate

$$
\begin{aligned}
J_{2} & =-\int_{\left\{\frac{1}{2} \leq|x| \leq 2\right\}} \int_{\left\{|y| \leq \frac{1}{4}\right\}} u(x, t) u(y, t) \frac{x-y}{|x-y|^{2}} \cdot \mu(x) \mathrm{d} x \mathrm{~d} y \\
& \geq-4 \varrho M \int_{\left\{\frac{1}{2} \leq|x| \leq 2\right\}} \Delta \Phi(x) u(x, t) \mathrm{d} x-C_{\varrho} M \int_{\left\{\frac{1}{2} \leq|x| \leq 2\right\}} \Phi(x) u(x, t) \mathrm{d} x
\end{aligned}
$$

where we put $\varrho=\frac{1}{24 M}, A=6 C M$, and we obtain the required inequality.

To exploit some gain from the symmetry assumption (1.8) (i.e. discover some cancellations in the integral $J_{3}$ ), we decompose $J_{3}$ further into the integrals $J_{3,1}$ and $J_{3,2}$ over the disjoint sets

$\Omega_{1}=\left\{\frac{1}{4} \leq|x| \leq A, \frac{1}{4} \leq|y| \leq A, x \in \Gamma_{y}\right\}$ and $\Omega_{2}=\left\{\frac{1}{4} \leq|x| \leq A, \frac{1}{4} \leq|y| \leq A, x \notin \Gamma_{y}\right\}$,

where $\Gamma_{y}=\left\{x:\left|\frac{x}{|x|}-\frac{y}{|y|}\right| \leq \frac{2 \pi}{N}\right\}$ is the sector determined by the direction of $y$. Obviously, we have $x \in \Gamma_{y} \Leftrightarrow y \in \Gamma_{x}$. Denote by $S \subset \mathbb{R}^{4}$ the support of the function $|\mu(x)|+|\mu(y)|$. Since $\Omega_{1}$ is symmetric and if $(x, y) \in S$ then either $\frac{1}{2}<|x|<2$ or 
$\frac{1}{2}<|y|<2$, so for the first integral we have the representation

$$
\begin{aligned}
J_{3,1} & =\iint_{\Omega_{1}} u(x, t) u(y, t) \frac{x-y}{|x-y|^{2}} \cdot \mu(x) \mathrm{d} x \mathrm{~d} y \\
& =\frac{1}{2} \iint_{\Omega_{1}} u(x, t) u(y, t) \frac{(x-y) \cdot(\mu(x)-\mu(y))}{|x-y|^{2}} \mathrm{~d} x \mathrm{~d} y \\
& =\frac{1}{2} \iint_{\Omega_{1} \cap S} u(x, t) u(y, t) \frac{(x-y) \cdot(\nabla \Phi(x)-\nabla \Phi(y))}{|x-y|^{2}} \mathrm{~d} x \mathrm{~d} y .
\end{aligned}
$$

Therefore, we can estimate $\left|J_{3,1}\right|$ by

$$
\begin{aligned}
\left|J_{3,1}\right| & \leq 2 \int_{\left\{\frac{1}{4} \leq|x| \leq A, \frac{1}{4} \leq|y| \leq A, x \in \Gamma_{y}\right\} \cap S} u(x, t) u(y, t) \mathrm{d} x \mathrm{~d} y \\
& \leq C \frac{4}{N} M \int_{\left\{\frac{1}{2} \leq|x| \leq 2\right\}} u(x, t) \mathrm{d} x, \\
& \leq C \frac{4}{N} M\left(\int_{\left\{\frac{1}{2} \leq|x| \leq 2\right\}} u \Delta \Phi+C \int_{\left\{\frac{1}{2} \leq|x| \leq 2\right\}} u \Phi\right),
\end{aligned}
$$

since

$$
\int_{\left\{\frac{1}{4} \leq|y| \leq 2, y \in \Gamma_{x}\right\}} u(y, t) \mathrm{d} y \leq C \frac{2}{N} M \quad \text { for } x \in \operatorname{supp} \mu,
$$

due to the $N$-symmetry assumption.

For the estimate of the integral $J_{3,2}$, note that the weight function $\mu$ satisfies

$$
\frac{|(x-y) \cdot(\mu(x)-\mu(y))|}{|x-y|^{2}} \leq \frac{|\mu(x)-\mu(y)|}{|x-y|} .
$$

Moreover, we have

$$
|\mu(x)-\mu(y)| \leq C|x-y|, \quad|\mu(x)-\mu(y)| \leq|\mu(x)|+|\mu(y)|,
$$

so putting together those inequalities, we arrive at

$$
|\mu(x)-\mu(y)| \leq C|x-y|^{\frac{1}{2}}\left(|\mu(x)|^{\frac{1}{2}}+|\mu(y)|^{\frac{1}{2}}\right) .
$$

Observe that, if $x \notin \Gamma_{y},|x|,|y| \geq \frac{1}{2}$, then $|x-y| \geq \frac{1}{c N}$ for some $c>0$. Consequently, using the symmetry of $\Omega_{2}$, we obtain after splitting the integration domain into dyadic pieces

$$
\begin{aligned}
\left|J_{3,2}\right| & \leq C \iint_{\left\{\frac{1}{2} \leq|x| \leq A, \frac{1}{4} \leq|y| \leq A, x \notin \Gamma_{y}\right\}}|x-y|^{-\frac{1}{2}}\left(|\mu(x)|^{\frac{1}{2}}+|\mu(y)|^{\frac{1}{2}}\right) u(x, t) u(y, t) \mathrm{d} x \mathrm{~d} y \\
& \leq C \sum_{1 \leq L \leq c N, \text { dyadic }}\left(\frac{L}{N}\right)_{\left\{\frac{1}{2} \leq|x| \leq A, \frac{1}{4} \leq|y| \leq A, x \notin \Gamma_{y},|x-y| \simeq \frac{L}{N}\right\}} \iint_{\mid}\left(|\mu(x)|^{\frac{1}{2}}+|\mu(y)|^{\frac{1}{2}}\right) u(x, t) u(y, t) \mathrm{d} x \mathrm{~d} y
\end{aligned}
$$


with a constant $C>0$.

Observe that by the $N$-symmetry property, for $\frac{1}{2} \leq|x| \leq 2$, we have the bound

$$
\int_{\left\{|y| \geq \frac{1}{4},|x-y| \simeq \frac{L}{N}\right\}} u(y, t) \mathrm{d} y \leq C \frac{L}{N} M .
$$

Applying this, we estimate each summand by

$$
2 C L^{-\frac{1}{2}} N^{\frac{1}{2}} \int|\mu(x)|^{\frac{1}{2}} u(x, t) \mathrm{d} x M \frac{L}{N}=C M L^{\frac{1}{2}} N^{-\frac{1}{2}} \int|\mu(x)|^{\frac{1}{2}} u(x, t) \mathrm{d} x .
$$

Since $\sum_{1 \leq L \leq c N \text {,dyadic }} L^{\frac{1}{2}} \simeq c N^{\frac{1}{2}}$, the entire sum is bounded from above by an application of inequality (3.22)

$$
C M \int|\mu(x)|^{\frac{1}{2}} u(x, t) \mathrm{d} x \leq C \varrho M \int_{\left\{\frac{1}{2} \leq|x| \leq 2\right\}} \Delta \Phi(x) u(x, t) \mathrm{d} x+C_{\varrho} M \int_{\left\{\frac{1}{2} \leq|x| \leq 2\right\}} \Phi(x) u(x, t) \mathrm{d} x .
$$

Now, we put $\rho=\frac{1}{6 C M}$ and adding the inequalities we obtain the desired estimate $-\left|I_{1, \mu}\right| \geq-\frac{1}{2} \int_{\left\{\frac{1}{2} \leq|x| \leq 2\right\}} \Delta \Phi u-C \int_{\left\{\frac{1}{2} \leq|x| \leq 2\right\}} \Phi u$.

Similar considerations apply to the part containing the function $\nu$ in the decomposition (3.19), and we get $-\left|I_{1, \nu}\right| \geq-\frac{1}{2} \int_{\{R \leq|x| \leq 3 R\}} \Delta \Phi u-C R^{-2} \int_{\{R \leq|x| \leq 3 R\}} \Phi u$. We note that for $R=4$ the proof is literally as the above, and the general case $R \geq 4$ follows by scalling. Adding this inequalities, and using $\Delta \Phi(x)=0$ for $2 \leq|x| \leq R$, we obtain the differential inequality

$$
\frac{\mathrm{d}}{\mathrm{d} t} H(t) \geq-C H(t)
$$

similarly as was in (3.18). Note that we established that the assumption $N \geq C M$ where $C$ is large enough (but it does not depend on $M$ ) is sufficient in order to Proposition 3.9 holds for $\delta=1$.

To complete the proof for arbitrary $0<\delta \leq 1<R$, it suffices to use the rescaling defined in (1.9) with $\lambda=1 / \delta$, and then to replace $\delta R$ by $R$.

\subsection{Proofs of the $8 \pi$-mass concentration results.}

Proof of Theorem 1.3. The idea of the proof is easy. Suppose for contradiction that $\int_{\left\{|x| \leq R_{j}\right\}} u\left(x, t_{j}\right) \mathrm{d} x \geq 8 \pi+\varepsilon$ for some sequences $t_{j} \nearrow T_{\mathrm{b}}, R_{j} \searrow 0$, and a positive 
$\varepsilon>0$. It suffices to construct a sequence $\left\{S_{j}\right\}$ such that

$$
\int_{\left\{S_{j}<|x| \leq 3 R_{j}\right\}} u(x, t) \mathrm{d} x \geq \eta(\varepsilon)>0 \quad \text { for all } t \in\left[t_{j}, T_{\mathrm{b}}\right),
$$

where $\eta(\varepsilon)>0$ is independent of $j$. If the annuli $\left\{x \in \mathbb{R}^{2}: S_{j}<|x|<R_{j}\right\}$ are disjoint, then the total mass $\int u(x, t) \mathrm{d} x$ tends to infinity as $t \rightarrow T_{\mathrm{b}}$, which is impossible.

Under the assumption that $\int_{\left\{|x| \leq R_{j}\right\}} u\left(x, t_{j}\right) \mathrm{d} x \geq 8 \pi+\varepsilon$ for a positive $\varepsilon>0$ and some sequences $t_{j} \nearrow T_{\mathrm{b}}, R_{j} \searrow 0$, there exists a sufficiently large constant $L$ depending only on $M$ and $\varepsilon$, such that we have $\int \psi_{L R_{j}}(x) u\left(x, t_{j}\right) \mathrm{d} x \geq 8 \pi+\frac{\varepsilon}{2}$. To simplify the notation, we will denote from now on the radii $L R_{j}$ again by $R_{j}$.

We also define a sequence $0<S_{j}<R_{j}$ such that $w_{S_{j}}\left(t_{j}\right)=8 \pi+\frac{\varepsilon}{4}$, while by our choice $w_{R_{j}}\left(t_{j}\right) \geq 8 \pi+\frac{\varepsilon}{2}$. Observe, that the rescaled function $u_{j}(x, t)=$ $S_{j}^{2} u\left(S_{j} x, t_{j}+S_{j}^{2} t\right)$ is a solution of the system (1.1)-(1.2) with the same mass $M$, cf. (1.9). Moreover, its initial condition $u_{j}(x, 0)=S_{j}^{2} u\left(S_{j} x, t_{j}\right)$ satisfies assumptions of Proposition 3.1, that is

$$
\int_{\{|x| \leq 1\}} u_{j}(x, 0) \mathrm{d} x \geq \int_{\{|x| \leq 1\}} \psi(x) u_{j}(x, 0) \mathrm{d} x \geq 8 \pi+\frac{\varepsilon}{4} .
$$

Hence, the solution $u_{j}(x, t)$ cannot be classical after (by the definition, it means that this blows up not later than) $T_{\mathrm{w}}$, where $T_{\mathrm{w}}=T_{\mathrm{w}}(M, \varepsilon)$ can be chosen independently of $j$ by Proposition 3.1. Denote by $T_{\mathrm{b}}(j) \leq T_{\mathrm{w}}(M, \varepsilon)$ the blowup time of the solution $u_{j}(x, t)$.

By the definition of $S_{j}$ and $R_{j}$ and (2.2), we have

$$
w_{R_{j}}\left(t_{j}\right)-w_{S_{j}}\left(t_{j}\right) \geq \frac{\varepsilon}{8} .
$$

Passing to the rescaled solution $u_{j}(x, t)$, we obtain

$$
\int\left(\psi_{\frac{R_{j}}{S_{j}}}(x)-\psi(x)\right) u_{j}(x, 0) \mathrm{d} x \geq \frac{\varepsilon}{16} .
$$

Since we have by (3.2) $\left|\psi_{\frac{R_{j}}{S_{j}}}(x)-\psi(x)\right| \leq C|x|^{2}$ for some $C=C(B)>0$, we obtain for an appropriate choice of the constant $\delta=\delta(M, \varepsilon)$ (it suffices to choose 
$\left.\delta^{2}(M, \varepsilon)=\varepsilon /(16 C M)\right)$, still independent of $j$,

$$
\int_{\left\{\delta \leq|x| \leq \frac{R_{j}}{S_{j}}\right\}} u_{j}(x, 0) \mathrm{d} x \geq \frac{\varepsilon}{16} .
$$

Applying Proposition 3.9 we infer that for $t \leq T_{\mathrm{b}}(j)$ we have

$$
\int_{\left\{\frac{1}{2} \delta \leq|x| \leq 3 \frac{R_{j}}{S_{j}}\right\}} u_{j}(x, t) \mathrm{d} x \geq \beta \frac{\varepsilon}{8},
$$

where the constant $\beta>0$ depends only on $T_{\mathrm{w}}(M, \varepsilon), M, \varepsilon$, and consequently

$$
\int_{\left\{\frac{1}{2} \delta \leq|x| \leq 3 \frac{R_{j}}{S_{j}}\right\}} u_{j}\left(x, T_{\mathrm{b}}(j)\right) \mathrm{d} x \geq \beta \frac{\varepsilon}{8}
$$

with the same constant $\beta$. Scaling back to the original coordinates, we get at the blowup time $T_{\mathrm{b}}$

$$
\int_{\left\{\frac{1}{2} \delta S_{j} \leq|x| \leq 3 R_{j}\right\}} u\left(x, T_{\mathrm{b}}\right) \mathrm{d} x \geq \beta \frac{\varepsilon}{8} .
$$

So, if we choose $R_{j+1}$ (passing, if necessary, to a subsequence) satisfying $3 R_{j+1} \leq$ $\frac{1}{2} \delta S_{j}$, so that the annuli $\left\{\frac{1}{2} \delta S_{j} \leq|x| \leq 3 R_{j}\right\}$ are disjoint, we infer that $u\left(x, T_{\mathrm{b}}\right)$ accumulates infinite mass. Indeed, masses estimated in (3.23) (each bounded from below by the same positive number) are distributed over disjoint annuli $\left\{\frac{1}{2} \delta S_{j} \leq\right.$ $\left.|x| \leq 3 R_{j}\right\}$ - a contradiction.

Proof of Corollary 1.4. First, recall the main result obtained in [8]: if the initial condition satisfies $\int_{B} u_{0}(x) \mathrm{d} x \leq 8 \pi-\varepsilon$ for every ball $B$ of radius $\delta$, then the solution of problem (1.1)-(1.3) exists at least on the interval $[0, T(M, \delta, \varepsilon)]$, where the number $T(M, \delta, \varepsilon)>0$ depends on $M, \delta, \varepsilon$, only.

Now, let $u$ be an $N$-symmetric solution with properties assumed in Theorem 1.3. Suppose that, for some fixed $\varepsilon>0$ and $\delta>0$, there exists a sequence $t_{j} \nearrow T_{\mathrm{b}}$ such that

$$
\int_{\{|x| \leq \delta\}} u\left(x, t_{j}\right) \mathrm{d} x \leq 8 \pi-\varepsilon \quad \text { for each } t_{j} .
$$


Using the $N$-symmetry property and (3.24), it is easy to show that there exists $\delta_{1} \in(0, \delta]$ such that

$$
\int_{\left\{\left|x-x_{0}\right| \leq \delta_{1}\right\}} u\left(x, t_{j}\right) \mathrm{d} x \leq 8 \pi-\varepsilon \quad \text { for each } x_{0} \in \mathbb{R}^{2} \text { and each } t_{j}
$$

(use the $N$-symmetry if $\left|x_{0}\right|>\delta / 2$ and (3.24) if $\left|x_{0}\right|<\delta$ ).

Now, by [8], the solution of problem (1.1)-(1.3) with the initial condition $u\left(x, t_{j}\right)$, $T_{\mathrm{b}}-t_{j}<T\left(M, \delta_{1}, \varepsilon\right) / 2$, exists at least on the interval $\left[0, T\left(M, \delta_{1}, \varepsilon\right) / 2\right]$. Thus, we have extended the solution beyond the blowup time $T_{\mathrm{b}}$ which is a contradiction.

Therefore, we have proved that

$$
\liminf _{t \rightarrow T_{\mathrm{b}}} \int_{\{|x|<\delta\}} u(x, t) \mathrm{d} x \geq 8 \pi \quad \text { for all } \delta>0,
$$

which, together with the upper bound in Theorem 1.3, completes the proof of this corollary.

\section{REFERENCES}

[1] J. Bedrossian, N. Masmoudi, Existence, uniqueness and Lipschitz dependence for PatlakKeller-Segel and Navier-Stokes in $\mathbb{R}^{2}$ with measure-valued initial data, Arch. Rational Mech. Anal. 214 (2014), 717-801.

[2] P. Biler, The Cauchy problem and self-similar solutions for a nonlinear parabolic equation, Studia Math. 114 (1995), 181-205.

[3] P. Biler, Existence and nonexistence of solutions for a model of gravitational interaction of particles III, Coll. Math. 68 (1995), 229-239.

[4] P. Biler, Radially symmetric solutions of a chemotaxis model in the plane - the supercritical case, 31-42, in: Parabolic and Navier-Stokes Equations, Banach Center Publications 81, Polish Acad. Sci., Warsaw, 2008.

[5] P. Biler, G. Karch, Blowup of solutions to generalized Keller-Segel model, J. Evol. Eq. 10 (2010), 247-262.

[6] P. Biler, T. Cieślak, G. Karch, J. Zienkiewicz, Local criteria for blowup of solutions in twodimensional chemotaxis models, arXiv: 1410.7807 v.2.

[7] P. Biler, G. Karch, J. Zienkiewicz, Morrey spaces norms and criteria for blowup in chemotaxis models, 1-13, to appear in: Networks and Heterogeneous Media (2015).

[8] P. Biler, J. Zienkiewicz, Existence of solutions for the Keller-Segel model of chemotaxis with measures as initial data, Bull. Pol. Acad. Sci. Math. 63 (2015), 41-51. DOI: 10.4064/ba63-1-6.

[9] A. Blanchet, J. Dolbeault, B. Perthame, Two-dimensional Keller-Segel model: optimal critical mass and qualitative properties of the solutions, Electron. J. Differential Equations 44, 32 pp. (2006).

[10] M. A. Herrero, E. Medina, J. J. L. Velázquez, Finite-time aggregation into a single point in a reaction-diffusion system, Nonlinearity 10 (1997), 1739-1754.

[11] M. Kurokiba, T. Ogawa, Finite time blow-up of the solution for a nonlinear parabolic equation of drift-diffusion type, Differ. Integral Eq. 16 (2003), 427-452.

[12] P.-G. Lemarié-Rieusset, Small data in an optimal Banach space for the parabolic-parabolic and parabolic-elliptic Keller-Segel equations in the whole space, Adv. Diff. Eq. 18 (2013), 1189-1208.

[13] T. Nagai, Blowup of nonradial solutions to parabolic-elliptic systems modeling chemotaxis in two-dimensional domains, J. Inequal. Appl. 6 (2001), 37-55. 
[14] S. Seki, Y. Sugiyama, J. J. L. Velázquez, Multiple peak aggregations for the Keller-Segel system, Nonlinearity 26 (2013), 319-352.

[15] T. Senba, T. Suzuki, Chemotactic collapse in a parabolic-elliptic system of mathematical biology, Adv. Diff. Eq. 6 (2001), 21-50.

[16] T. Suzuki, Free energy and self-interacting particles, Progress in Nonlinear Differential Equations and Their Applications, vol. 62, Birkhäuser, Boston, 2005.

Instytut Matematyczny, Uniwersytet Wroceawski, Pl. Grunwaldzki 2/4, 50-384 WrocŁaW, Poland

E-mail address: Piotr.Biler@math.uni.wroc.pl

Instytut Matematyczny, Uniwersytet WrocŁaWski, Pl. Grunwaldzki 2/4, 50-384 WrocŁaw, Poland

E-mail address: Grzegorz.Karch@math.uni.wroc.pl

$U R L:$ http://www.math.uni.wroc.pl/ ${ }^{\sim} k a r c h$

Instytut Matematyczny, Uniwersytet Wroceawski, Pl. Grunwaldzki 2/4, 50-384 WrocŁaW, POLAND

E-mail address: Jacek.Zienkiewicz@math.uni.wroc.pl 